\title{
Effect of graphite degradation on the LCF properties of nodular cast iron
}

\author{
Josef Denk
}

ABB Turbo Systems, Development Department ZTE, 5401 Baden, Switzerland

\begin{abstract}
The effect of degraded microstructure on the tensile and LCF properties was studied on a casing of a turbocharger, which exhibited locally irregular graphite formation. The tensile tests showed that the ductility values of the zone with degraded graphite decreased strongly compared to the zone with normal graphite, while the strength values decreased only slightly. Based on these results and a LCF reference curve for nodular cast iron with normal graphite structure, a synthetic LCF curve was generated for the material condition with degraded graphite structure. LCF tests on specimens with irregular graphite structure confirmed the predicted LCF behaviour quite good.
\end{abstract}

\section{Investigation}

The present investigation was carried out on a gas outlet casing of a large turbocharger, which was made nominally from nodular cast iron of the type GJS-400-15.

Locally an abnormal graphite structure was found during the quality assurance tests after machining. Figure 1 shows both types of microstructure in the flange of the casing and indicates that the irregular microstructure can be detected as grey zones already by visual inspection.

Samples for tensile and strain controlled fatigue tests (LCF tests) were taken from the zone with degraded microstructure and from a non-affected zone.

The tensile tests were carried out according to ISO 6892-1 and the LCF tests were performed according to ISO 12106. All tests took place at room temperature by using cylindrical specimens with threads.

\section{Results}

In Fig. 2 the results of the tensile tests are shown versus the distance from the outer rim of the flange. Close to this outer rim, i.e. in the zone with degraded graphite form, the fracture elongation was far below the specified limit of $15 \%$.

While the tensile strength of the specimens with irregular graphite form decreased by about $26 \%$ the yield strength dropped only slightly by about $6 \%$.

This is an Open Access article distributed under the terms of the Creative Commons Attribution License 4.0, which permits unrestricted use, distribution, and reproduction in any medium, provided the original work is properly cited. 


\section{MATEC Web of Conferences}

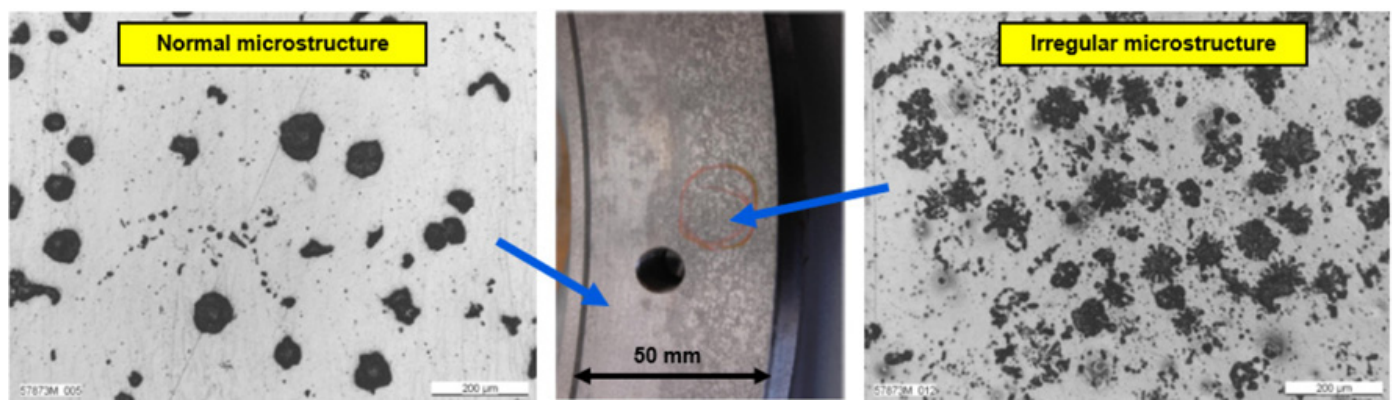

Figure 1. Normal and irregular microstructure in the flange of the nodular cast iron casing.
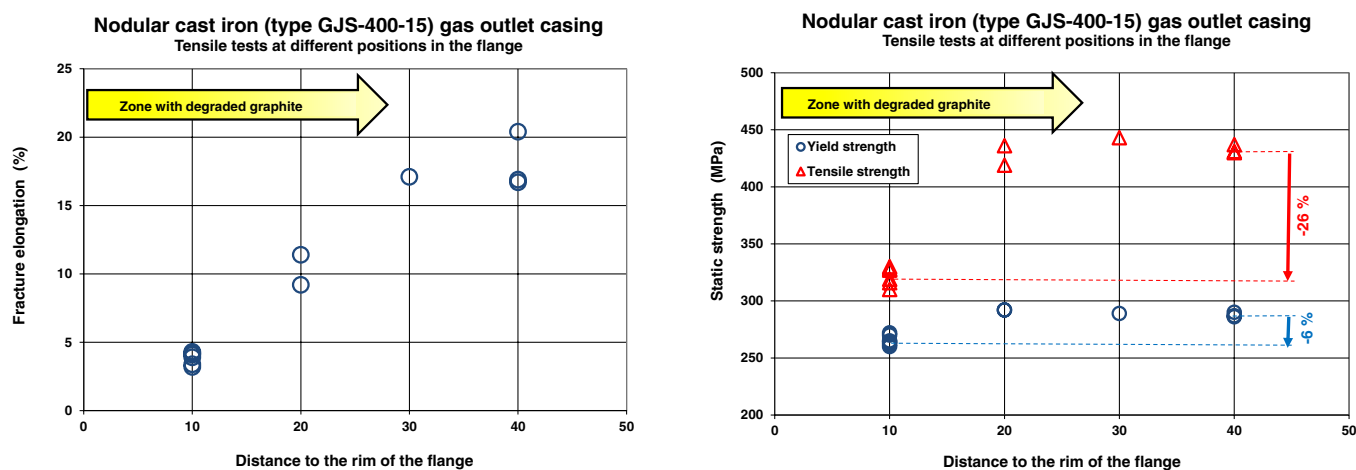

Figure 2. Fracture elongation and static strength versus the sampling position of the tensile specimens.

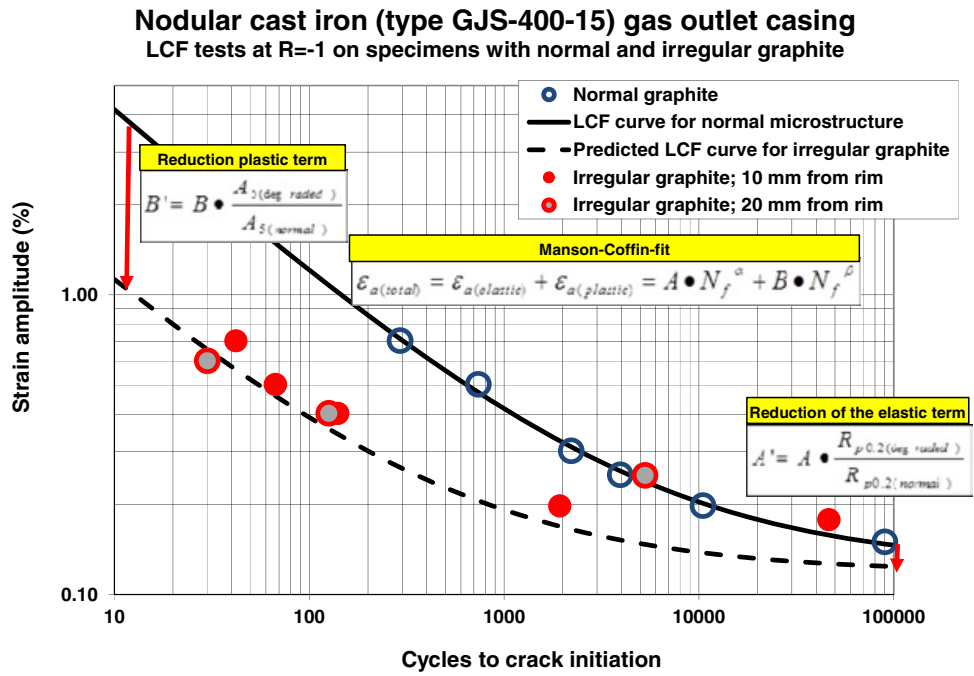

Figure 3. LCF tests on specimens with normal and irregular graphite structure.

Figure 3 shows the results of the LCF tests on specimens with normal microstructure and those from two positions in the flange with irregular graphite structure. The LCF data with normal graphite were fitted by a Manson-Coffin-function as illustrated in the graph. 
Based on the results of the tensile tests a fictitious LCF curve for the degraded microstructure was predicted. The factor $\mathrm{A}$ of the elastic term was reduced by the relation of the yield strength levels of degraded and normal microstructure and the factor B of the plastic term was reduced by the relation of the fracture elongations of degraded and normal microstructure. The subsequent LCF tests of specimens with irregular graphite confirmed this approach quite good.

\section{Conclusions}

The investigation showed that the LCF behaviour for nodular cast iron with irregular graphite structure and degraded mechanical properties can be predicted based on the results of tensile tests.

The dominant effect is the reduction of the ductility, which affects the plastic term of the LCF curve and reduces the cycles to crack initiation quite significant in the range of high over-elastic loading. The lower the loading the smaller is the difference between normal and irregular graphite structure. 